\title{
The Human Stratum Corneum Prevents Small Gold Nanoparticle Penetration and Their Potential Toxic Metabolic Consequences
}

\author{
David C. Liu, ${ }^{1,2}$ Anthony P. Raphael, ${ }^{1}$ Daniel Sundh, ${ }^{1,3}$ Jeffrey E. Grice, ${ }^{2}$ \\ H. Peter Soyer, ${ }^{1}$ Michael S. Roberts, ${ }^{2,4}$ and Tarl W. Prow ${ }^{1,2}$ \\ ${ }^{1}$ Dermatology Research Centre, School of Medicine, Princess Alexandra Hospital, The University of Queensland, Brisbane, \\ QLD 4102, Australia \\ ${ }^{2}$ Therapeutics Research Centre, School of Medicine, Princess Alexandra Hospital, The University of Queensland, Brisbane, \\ QLD 4102, Australia \\ ${ }^{3}$ Institute of Neuroscience and Physiology, The Sahlgrenska Academy, University of Gothenburg, 40530 Gothenburg, Sweden \\ ${ }^{4}$ School of Pharmacy and Medical Science, University of South Australia, Adelaide, SA 5001, Australia
}

Correspondence should be addressed to Tarl W. Prow, t.prow@uq.edu.au

Received 20 January 2012; Accepted 12 April 2012

Academic Editor: Xiaoming Li

Copyright (C) 2012 David C. Liu et al. This is an open access article distributed under the Creative Commons Attribution License, which permits unrestricted use, distribution, and reproduction in any medium, provided the original work is properly cited.

\begin{abstract}
Nanoparticles are being used in multiple applications, ranging from biomedical and skin care products (e.g., sunscreen) through to industrial manufacturing processes (e.g., water purification). The increase in exposure has led to multiple reports on nanoparticle penetration and toxicity. However, the correlation between nanoparticle size and its penetration without physical/chemical enhancers through the skin is poorly understood-with studies instead focusing primarily on skin penetration under disrupted conditions. In this paper, we investigate the penetration and metabolic effects of $10 \mathrm{~nm}, 30 \mathrm{~nm}$, and 60 nm gold nanoparticles within viable excised human skin after 24-hour exposure using multiphoton tomograph-fluorescence lifetime imaging microscopy. After 24 hour treatment with the 10,30, and $60 \mathrm{~nm}$ gold nanoparticles, there was no significant penetration detected below the stratum corneum. Furthermore, there were no changes in metabolic output (total NAD $(\mathrm{P}) \mathrm{H}$ ) in the viable epidermis posttreatment correlating with lack of penetration of nanoparticles. These results are significant for estimating topical nanoparticle exposure in humans where other model systems may overestimate the exposure of nanoparticles to the viable epidermis. Our data shows that viable human skin resists permeation of small nanoparticles in a size range that has been reported to penetrate deeply in other skin models.
\end{abstract}

\section{Introduction}

The skin is the largest organ of the body and is the primary barrier to nanoparticle exposure from naturally occurring and engineered nanomaterials found in the environment and workplace [1-3]. Therefore, it is important to understand the penetration characteristics of nanoparticles within the skin. However, accurate assessment of nanoparticle penetration is challenging, with results dependant on the model used (e.g., rat, pig, or human skin), barrier integrity (i.e., skin preparation prior to and during studies), nanoparticle properties (e.g., size, shape, surface structures, and charge), and degradation kinetics.

A number of reports have shown that nanoparticles penetrate skin into the dermis under various physically disrupted conditions [4-8]. In addition, nanoparticles have also been shown to penetrate skin in the presence of chemical enhancers [3]. We have previously shown ex vivo that skin treated with gold nanoparticles suspended in toluene results in nanoparticle penetration into the epidermis due to toluene acting as a chemical enhancer and disrupting the stratum corneum [8]. Even though there are multiple reviews on nanoparticle toxicity and penetration within the skin [9-13], the correlation between nanoparticle size and its penetration without physical/chemical enhancers is poorly understoodwith studies instead focusing primarily on skin penetration under disrupted conditions.

A recent report by Filon et al. (2011) showed $12.9 \mathrm{~nm}$ gold nanoparticle penetration in thawed human skin [14]. The results showed that gold nanoparticle penetration occurred after 24 hours of treatment in both intact and damaged skin. Gold nanoparticles were detected in the 
receiving solution of the Franz cells after 24 hours of skin exposure. This report supports a previous study conducted by Sonavane et al. (2008) where $15 \mathrm{~nm}$ gold nanoparticles penetrated through the excised rat skin into the dermis also after 24-hour treatment [15].

In the last 20 years, multiphoton tomography (MPT) has developed into a technique that enables the concurrent monitoring of both the organ morphology and solute transported into that organ, including the skin, with use increasing in clinical applications [16-18]. A benefit of MPT is that it can be used in conjunction with fluorescence lifetime imaging (FLIM). FLIM is a sensitive, time-resolved technique that enables the detection of the lifetimes and intensity of fluorescent molecules within the image plane [19]. MPT-FLIM is also used to monitor the autofluorescence of fluorophores within the skin including NAD/NADP (i.e., NAD $[\mathrm{P}] \mathrm{H}$ ), flavin adenine dinucleotide (FAD), and porphyrins. We have previously shown that FLIM can also be used to separate metal nanoparticle signals from skin autofluorescence [20]. However, the fluorescence lifetime decay curves of gold nanoparticles and $\mathrm{NAD}(\mathrm{P}) \mathrm{H}$ cannot directly be separated [8]. Instead, the photon intensity output based on the $\alpha \%$ parameters can be used to differentiate the gold nanoparticle and $\mathrm{NAD}(\mathrm{P}) \mathrm{H}$ signal.

In this paper, our aim is to investigate the relationship between gold nanoparticle size and its penetration into viable human skin following topical exposure without physi$\mathrm{cal} /$ chemical enhancers. Gold nanoparticles $(10 \mathrm{~nm}, 30 \mathrm{~nm}$, and $60 \mathrm{~nm}$ ) were applied to freshly excised human skin for 24 hours. Following application the penetration profile was analysed using microscopy of whole intact skin and histology sections. Changes in the metabolic state of viable skin following gold nanoparticle treatment were evaluated by analysing the change in $\mathrm{NAD}(\mathrm{P}) \mathrm{H}$ using MPT-FLIM. Our data showed that there was no significant penetration of the different sized nanoparticles into the viable tissue even after 24 hours of treatment. Instead, the nanoparticles were located on the skin surface, within the stratum corneum and within the skin furrows. There were also no significant metabolic perturbations associated with gold nanoparticle treatment due to their lack of penetration. Overall, the data show that excised viable human skin is important for an accurate and reliable understanding of nanoparticle penetration.

\section{Materials and Methods}

2.1. Materials. Three different sized gold nanoparticles (10 nm [21], $30 \mathrm{~nm}$ [22], and $60 \mathrm{~nm} \mathrm{[23])} \mathrm{suspended} \mathrm{in} 5 \mathrm{~mL}$ sodium citrate were prepared and purchased from National Institute of Standards and Technology (NIST) of USA. The concentration of gold nanoparticles was $5 \mathrm{mg} / \mathrm{mL}$. The gold nanoparticles were characterized by NIST in relation to their size and chemical and electrochemical properties $(10 \mathrm{~nm}$ [21], $30 \mathrm{~nm}$ [22], and $60 \mathrm{~nm} \mathrm{[23]).} \mathrm{The} \mathrm{particle} \mathrm{size} \mathrm{and}$ morphology were determined by atomic force microscopy (AFM), scanning electron microscopy (SEM), transmission electron microscopy (TEM), electrospray-differential mobility analysis (ES-DMA), dynamic light scattering (DLS), and small-angle X-ray scattering (SAXS). The mean size range varied slightly between the techniques, resulting in the average diameter $\pm 95 \% \mathrm{CI}$ :

$10 \mathrm{~nm}$ particles: $8.5 \pm 0.3 \mathrm{~nm}(\mathrm{AFM}), 9.9 \pm 0.1 \mathrm{~nm}$ (SEM), 8.9 $\pm 0.1 \mathrm{~nm}$ (TEM), $11.3 \pm 0.1 \mathrm{~nm}$ (ES-DMA), $13.5 \pm 0.1 \mathrm{~nm}(\mathrm{DLS})$, and $9.1 \pm 1.8 \mathrm{~nm}(\mathrm{SAXS})$.

$30 \mathrm{~nm}$ particles: $24.9 \pm 1.1 \mathrm{~nm}($ AFM), $26.9 \pm 0.1 \mathrm{~nm}$ $(\mathrm{SEM}), 27.6 \pm 2.1 \mathrm{~nm}(\mathrm{TEM}), 28.4 \pm 1.1 \mathrm{~nm}$ (ESDMA)), $26.5 \sim 28.6 \pm 0.9 \sim 3.6 \mathrm{~nm}$ (DLS), and $24.9 \pm 1.2 \mathrm{~nm}(\mathrm{SAXS})$.

$60 \mathrm{~nm}$ particles: $55.4 \pm 0.3 \mathrm{~nm}(\mathrm{AFM}), 54.9 \pm 0.4 \mathrm{~nm}$ (SEM), $56.0 \pm 0.5 \mathrm{~nm}(\mathrm{TEM}), 56.3 \pm 1.5 \mathrm{~nm}$ (ESDMA), $55.3 \sim 56.6 \pm 1.4 \sim 8.3 \mathrm{~nm}$ (DLS), and $53.2 \pm 5.3 \mathrm{~nm}(\mathrm{SAXS})$.

The shape of the gold nanoparticles was determined by SAXs with each measurement calculated from the mean primary component peak value by fitting the $I(Q)$ data resulting in a Q-range: $0.01 \AA-1$ to $2.0 \AA-1(10 \mathrm{~nm}), 0.0001 \AA$ 1 to $1 \AA-1(30 \mathrm{~nm})$, and $0.01 \AA-1$ to $2.0 \AA-1(60 \mathrm{~nm})$. Particle aggregation within the sodium citrate buffer was determined by SEM images and analysed with ImageJ v1.37. The data analysis of the Otsu threshold algorithm was produced from the binary image, distinguishing particles from the background. A total of $140(10 \mathrm{~nm}), 1185$ (30 nm), and 425 $(60 \mathrm{~nm})$ particles were analysed within an area of roughly $35 \times 35$ pixels $(10 \mathrm{~nm}), 50 \times 50$ pixels $(30 \mathrm{~nm}), 100 \times 100$ pixels $(60 \mathrm{~nm})$ from $2(10 \mathrm{~nm}), 3(30 \mathrm{~nm})$, and $3(60 \mathrm{~nm})$ randomly prepared ampoules.

All other chemicals and solvents were of reagent grade.

2.2. Excised Human Skin Preparation. Human skin was donated from abdominoplasty patients with the approval of the Princess Alexandra Hospital Research Ethics Committee (no. 1997/097). A total of three skin donors were used in this study. The donor skin was obtained on the day of removal from the patient, and the experiment was performed within 24 hours to preserve the viability of the skin. The subcutaneous fatty tissue was removed from the skin using a scalpel prior to the start of the experiments. The surface of skin was cleaned with deionised water, and the excised tissue was stored covered on saline moistened gauze in a $4^{\circ} \mathrm{C}$ cold room until use. Skin handling and experimental design was conducted to maintain metabolic functionality throughout the course of these studies [24].

2.3. Skin Penetration Studies. The excised skin was cut into $25 \mathrm{~mm}$ diameter skin discs and mounted in a Franz diffusion cell with a diffusion area of $1.1 \mathrm{~cm}^{2}$ and a receptor chamber volume of $3.2 \mathrm{~mL}$. High vacuum grease (Ajax, FineChem) was used to seal the edges of the two chambers. Phosphate buffered saline, $\mathrm{pH}$ 7.4, was then pipetted into the receptor chamber and magnetically stirred at $500 \mathrm{rpm}$ in a water bath at $35^{\circ} \mathrm{C}$. Prior totrans-epidermal water loss (TEWL) analysis the combined skin/Franz cell was equilibrated for 30 
minutes. TEWL was then measured using a Biox AquaFlux condenser chamber unit (model no. AF200) to evaluate the surface integrity of the skin surface prior to nanoparticle treatment [8]. After confirmation of intact viable skin, $500 \mu \mathrm{L}$ of $5 \mathrm{mg} / \mathrm{mL}$ gold nanoparticle suspensions was topically applied to the skin ( $n=3$ per group). The two control groups were topical application of saline and sodium citrate solution, respectively. The Franz diffusion cells were maintained at $35^{\circ} \mathrm{C}$ throughout the 24 -hour exposure period. After treatment, any excess gold nanoparticle suspension was drained and the skin removed. The treated skin was then dried at ambient conditions, and the TEWL was analysed again to determine any detrimental effect to the stratum corneum during treatment.

2.4. MPT-FLIM of Viable Treated Skin. Non-invasive microscopy analysis was conducted using MPT-FLIM with a DermaInspect (JenLab GmbH, Jena, Germany) equipped with time-correlated single-photon counting, SPC 830 FLIM detector (Becker \& Hickl, Berlin, Germany). The excitation source consisted of an ultrashort (65-femtosecond pulse width) pulsed mode-locked tuneable laser at $80 \mathrm{MHz}$ (Mai Tai, Spectra physics, Mountain View, USA), with a wavelength range of 710-920 nm. MPT-FLIM was used to simultaneously image the gold nanoparticles and endogenous $\mathrm{NAD}(\mathrm{P}) \mathrm{H}$ using an excitation wavelength of $740 \mathrm{~nm}$ [10]. The skin was optically sectioned from the stratum corneum to the stratum basale in $5 \mu \mathrm{m}$ increments using a $40 \mathrm{x}$ objective lens with an acquisition size image size of $210 \times$ $210 \mu \mathrm{m}^{2}$. The image was scanned at 13.4 seconds per frame at 600 zoom of region of interest (ROI). The emission filter used was a 350-650 nm bandpass filter (BG39) whith a long wavelength cut-off of $<700 \mathrm{~nm}$. A constant incident optical excitation power of $30 \mathrm{~mW}$ at the rear of the objective was used throughout the experiment. Three replicates of treated skin were analysed after 24 hours of treatment. FLIM data were analysed with a SPC 8302.9 Image software (Becker and Hickl) to generate fluorescence lifetime decay and photon contribution curves from $\mathrm{NAD}(\mathrm{P}) \mathrm{H}$ autofluorescence in the skin and gold luminescence. The instrument response function of each FLIM image of treated skin was calibrated to a sucrose crystal standard (Ajax Finechem Pty Ltd.). Each FLIM image was calculated using the "Decay matrix" command, and a selected ROI was cropped and analysed pixel by pixel and line by line in the selected fit parameter shown in a lifetime distribution histogram. Gold nanoparticle multiphoton enhanced photoluminescence was quantified using relative amplitude of pixel intensity/pixel frequency signal $\alpha_{1} \%$ from 95 to $100 \%$, and metabolic changes were quantified using $\alpha_{1} / \alpha_{2}$ ratio and total $\mathrm{NAD}(\mathrm{P}) \mathrm{H}$ lifetime intensity. The $\alpha_{1} \%$ is defined as the quantity of emission photons that return to the detector during the short lifetime decay curve phase, and the $\alpha_{2} \%$ is the long lifetime phase. The $\alpha_{1} \%$ is not affected by the microenvironment of the skin. The total $\mathrm{NAD}(\mathrm{P}) \mathrm{H}$ lifetime intensity is the combined total of free and bound $\mathrm{NAD}(\mathrm{P}) \mathrm{H}$ signals and was quantified using exported grey-scale image of each FLIM image with photons per pixel. The grey-scale image was imported to
ImageJ $1.43 \mathrm{u}$ (National Institutes of Health, USA) at 8-bit image quality and a selected cell area of $200 \times 200 \mu \mathrm{m}^{2}$. The selected cell area was measured, and the total NAD $(\mathrm{P}) \mathrm{H}$ mean intensity was quantified.

2.5. Cryosectioning of Treated Skin. Treated skin was fixed in $2 \%$ paraformaldehyde in $0.1 \mathrm{M}$ phosphate buffer, $\mathrm{pH} 7.4$, for 2 hours at room temperature. After fixation, the treated skin was embedded in OCT (Sakura Tissue-Tek) in a mold block and frozen at $70^{\circ} \mathrm{C}$ for 30 minutes. The tissue samples were sectioned at $10 \mu \mathrm{m}$ at $-25^{\circ} \mathrm{C}$ using a Leica CM1850 cryostat (Leica microsystems, Heidelberger, Nussloch, Germany). The sections were mounted in Prolong Gold (Invitrogen, Mulgrave, Victoria, Australia) prior to MPT-FLIM imaging.

2.6. Statistical Analysis. The differences in the measured macroscopic parameters between treated and control groups were compared by applying the nonparametric MannWhitney U-test. Two-way ANOVA analysis was used to determine the significance between groups. All calculations were done using Graphpad Prism version 5.03 (GraphPad software, Inc., USA) analysis. The level of significance was accepted at $P \leq 0.05$.

\section{Results}

3.1. Surface Barrier Integrity of Excised Viable Human Skin. Prior to and after treatment, the integrity of the stratum corneum (surface barrier) was analysed using transepidermal water loss (TEWL). The mean \pm SE of the TEWL was $30.6 \pm$ $4.1,28.2 \pm 1.2,30.1 \pm 0.9,28.9 \pm 1.4$, and $27.9 \pm 1.1$ for the saline, sodium citrate, $10 \mathrm{~nm}, 30 \mathrm{~nm}$, and $60 \mathrm{~nm}$ gold nanoparticle groups respectively, before treatment. After 24hour treatment, the TEWL had increased an average of 1.6, $1.8,1.8,1.5$ and 1.7 times compared to the pre-treatment values for saline, sodium citrate, $10 \mathrm{~nm}, 30 \mathrm{~nm}$ and $60 \mathrm{~nm}$ gold-nanoparticle-treated groups, respectively. There were no significant differences between any two groups within a single time point. However, there was a significant increase in TEWL for all groups after treatment.

3.2. Gold Nanoparticle Penetration into Skin. To determine the effect on penetration of the different sized nanoparticles, 10,30 , and $60 \mathrm{~nm}$ gold nanoparticles were topically applied to the skin for 24 hours followed by MPT and MPT-FLIM analysis. Two aspects of nanoparticle penetration within human skin were studied using MPT. The first consisted of en face analysis of whole intact human skin (Figure 1) and the second analysis of cryosectioned skin (Figure 2). Both sets of data (Figures 1 and 2) were pseudocoloured based on $\alpha_{1} \% 0-100 \%$ (blue to red). The autofluorescent components of living skin, that is, $\mathrm{NAD}(\mathrm{P}) \mathrm{H}$ and keratin result in $\alpha_{1} \%$ less than 95\% and are subsequently pseudocoloured green, blue, and yellow (viable skin only) [8]. The gold nanoparticle luminescence result in high $\alpha_{1} \%(95-100 \%)$ and is pseudocoloured orange to red. To determine any metabolic effects from the nanoparticles MPT-FLIM analysis was used to monitor $\mathrm{NAD}(\mathrm{P}) \mathrm{H}$. 


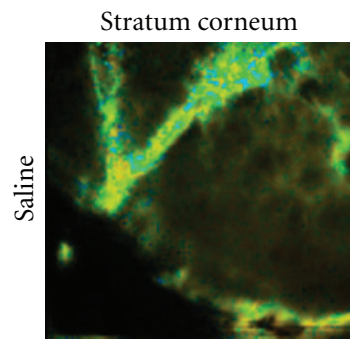

(a)



(e)

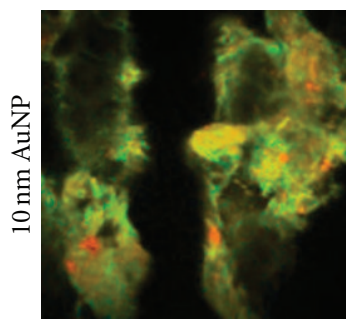

(i)

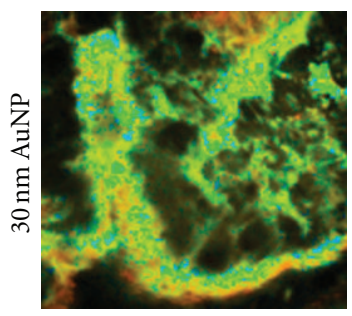

(m)

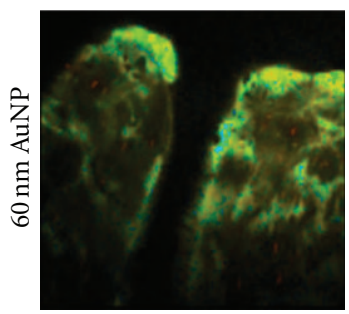

(q)

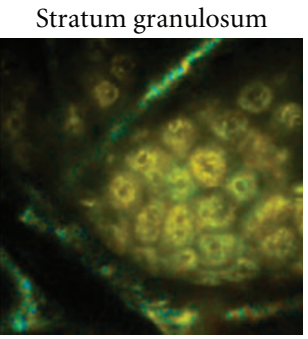

(b)

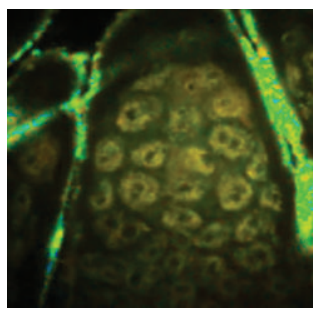

(f)



(j)

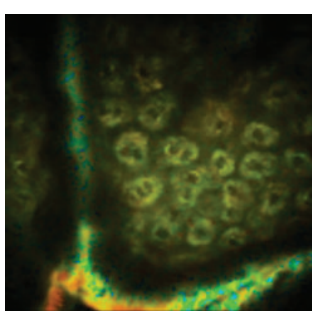

(n)

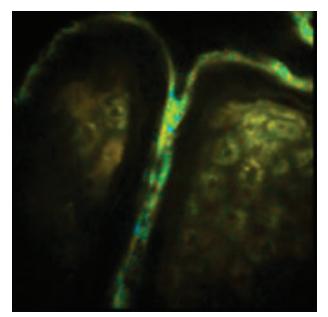

(r)

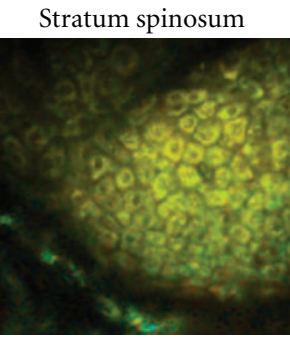

(c)



(g)

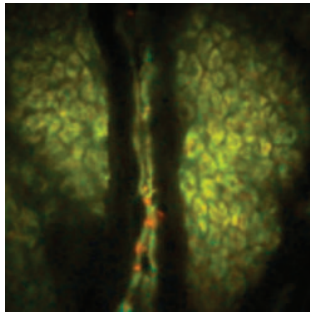

(k)

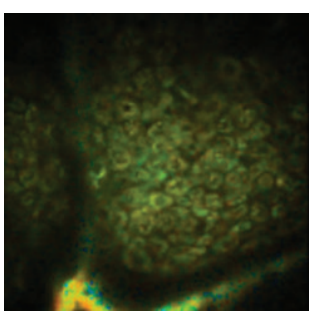

(o)

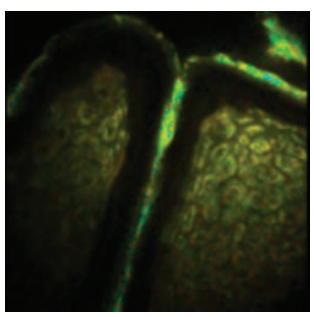

(s)

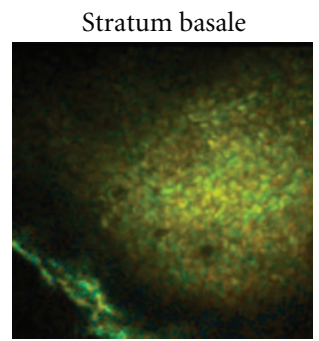

(d)

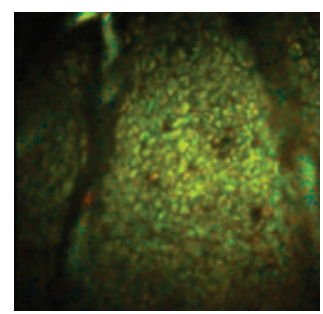

(h)

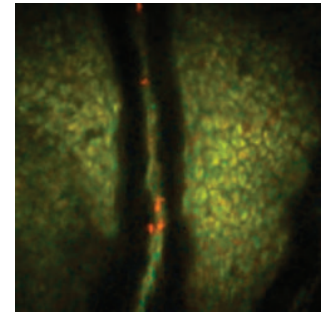

(1)

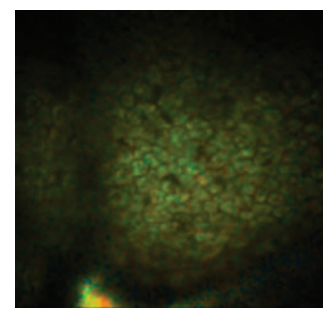

(p)

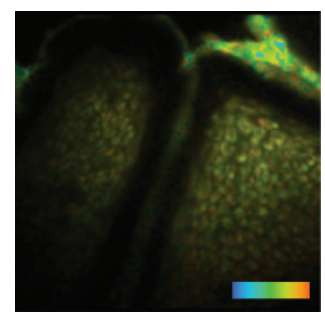

$(\mathrm{t})$

FIGURE 1: En face MPT-FLIM images of treated human viable skin. Multiphoton tomography fluorescence lifetime microscopy (FLIM) images showing the layers of excised full-thickness skin (stratum corneum, granulosum, spinosum, and basale) after 24-hour treatment with gold nanoparticles. Images are pseudocoloured based on $\alpha_{1} \%$ signal (skin autofluorescence 0-95\% and AuNPs 95-100\% signal). No AuNP signal was detected in the saline control (a)-(d); however, deposits of high signal were detected in the stratum corneum of the sodium citrate control (sodium citrate crystal aggregates).The AuNP-treated groups, $10 \mathrm{~nm}(\mathrm{i})-(1), 30 \mathrm{~nm}(\mathrm{~m})-(\mathrm{p})$, and $60 \mathrm{~nm}(\mathrm{q})$-(t), resulted in signal throughout the stratum corneum. $10 \mathrm{~nm}$ AuNPs were also detected in the furrows. Scale bar indicates $50 \mu \mathrm{m}$. Color bar, blue to red indicates $\alpha_{1} \% \quad 0-100 \%$. 


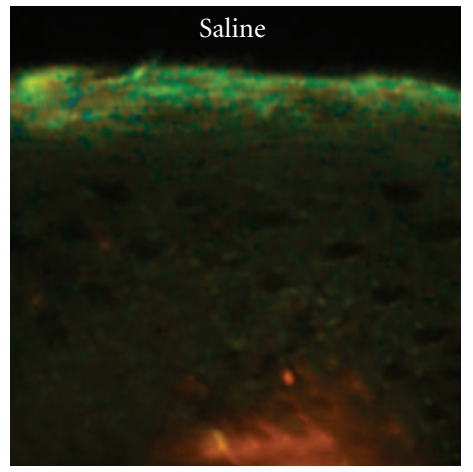

(a)



(b)

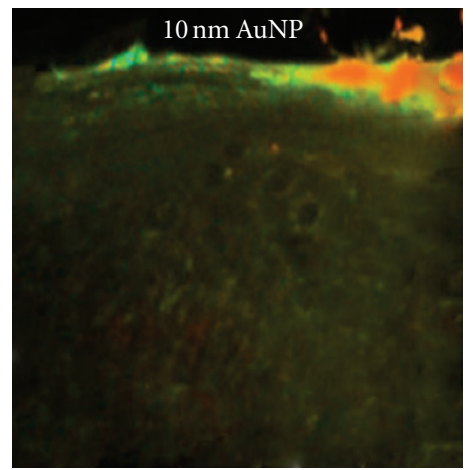

(c)

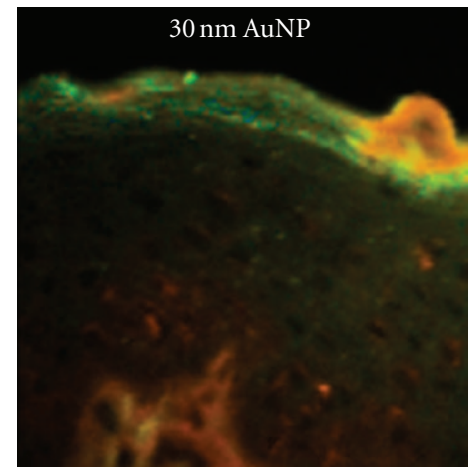

(d)

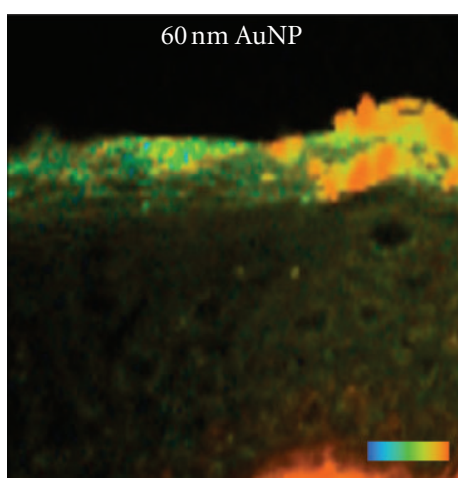

(e)

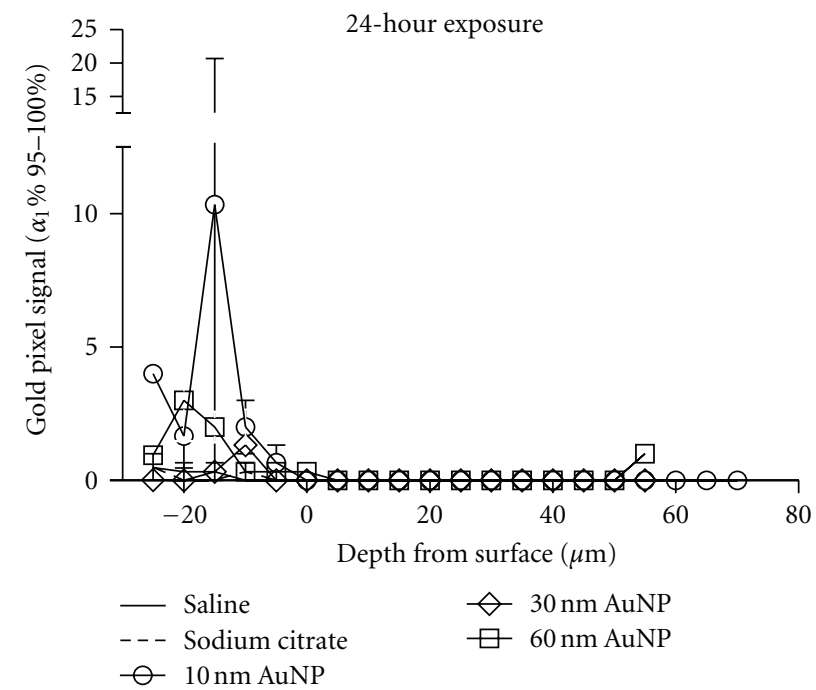

(f)

FIgURE 2: (a)-(e) Gold nanoparticle penetration in cryosectioned skin using MPT-FLIM and (f) gold nanoparticle luminescence skin depth profile. Cross-sectional images of the skin showing the superficial stratum corneum, viable epidermis and upper dermis. Images are pseudocoloured based on $\alpha_{1} \%$ signal (skin autofluorescence $0-95 \%$ and AuNPs $95-100 \%$ signal). Both the saline (a) and sodium citrate buffer (b) controls show no AuNP signal. The $10 \mathrm{~nm}$ (c), $30 \mathrm{~nm}$ (d), and $60 \mathrm{~nm}$ (e) AuNP-treated groups resulted in high amounts of AuNP signal in the stratum corneum with no signal in the lower layers. High signal was detected in the upper dermis corresponding to second harmonic generation of dermal collagen. Scale bar indicates $50 \mu \mathrm{m}$. Data from MPT-FLIM depth series (stratum corneum to basale of excised human skin) were processed to quantify gold nanoparticle luminescence intensity as a function of depth in non-furrow containing regions (i.e., AuNP penetration) (f). The top of the skin is at $-20 \mu \mathrm{m}$, whereas $0 \mu \mathrm{m}$ is the start of viable epidermis (stratum granulosum). These data were stacked from a depth series of en face images. Color bar, blue to red indicates $\alpha_{1} \% 0-100 \%$. 


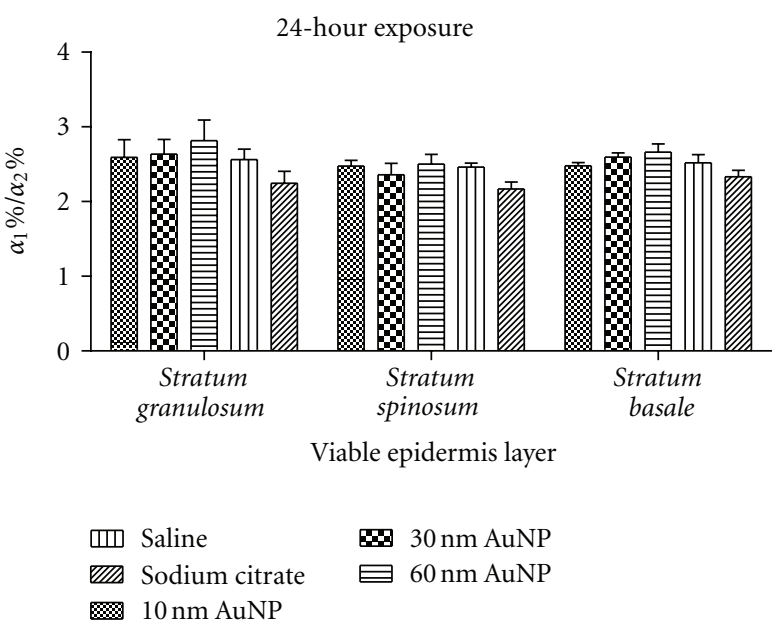

(a)

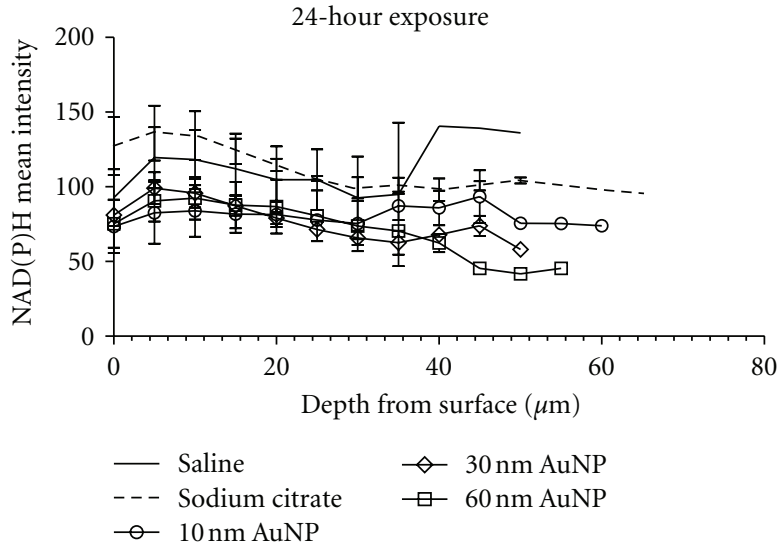

(b)

FIGURE 3: NAD(P)H effects from MPT-FLIM imaging. To assess the metabolic impact of AuNP penetration, NAD $(\mathrm{P}) \mathrm{H}$ signals were analysed using $\alpha_{1} \% / \alpha_{2} \%$ ratio and total NAD(P)H lifetime intensity using MPT-FLIM. The $\alpha_{1} \% / \alpha_{2} \%$ ratio (a) is inversely related to the metabolic rate and was calculated for each of the treatment groups at 24 hours from stratum granulosum, spinosum, and basale. The total NAD $(\mathrm{P}) \mathrm{H}$ lifetime intensities of selected area (b) were examined as a function of depth in non-furrow-containing regions. The beginning of viable epidermis is at $0 \mu \mathrm{m}$.

3.3. En Face MPT Analysis of Gold-Nanoparticle-Treated Skin. Initial analysis of nanoparticle penetration consisted of qualitative MPT analysis of whole intact human skin (Figure 1 $(n=3$ per group)). The skin was optically sectioned at $5 \mu \mathrm{m}$ intervals from the stratum corneum through the stratum granulosum, stratum spinosum and down to the base of the stratum basale. To determine if there was any background noise associated with the nanoparticle buffers, both saline and sodium citrate were applied to the skin in separate experiments and analysed-resulting in low background signal in all of the layers (Figures 1(a)-1(h)). Skin treated with the $10 \mathrm{~nm}$ gold nanoparticles resulted in a significant increase in high $\alpha_{1} \%$ signals throughout the stratum corneum compared to the controls (Figure 1(i)). There was no detection of AuNP signal in the stratum granulosum, spinosum, or basale. However, AuNP signal was detected on the surface within the furrows (Figures $1(j)-1(1)$ ). Both the 30 and $60 \mathrm{~nm}$ gold nanoparticle groups showed similar penetration results to the $10 \mathrm{~nm}$ nanoparticles with AuNP signal detected only in the stratum corneum (Figures $1(\mathrm{i})-1(\mathrm{t})$ ).

3.4. Cross-Sectional Analysis of Gold Nanoparticle Penetration within Skin. A second method using MPT analysis of cryosectioned skin was used to qualitatively assess the gold nanoparticle penetration. Representative images of each group are shown in Figure 2 resulting in a cross-sectional image of the stratum corneum (surface) down to the dermis. The control groups (Figures 2(a) and 2(b)) correlate with the results observed in Figure 1, with no AuNP signal detected within the upper layers of the skin. However, $\alpha_{1} \%$ (95$100 \%)$ signal was detected within the dermis of not only the control groups but also the nanoparticle-treated samples (Figures 2(a), 2(b), 2(d), and 2(e)). This signal is attributed to second harmonic generation of dermal collagen. The gold-nanoparticle-treated groups also correlate with the data observed in Figure 1, with AuNP signal detected only in the stratum corneum for all samples (Figures 2(c), 2(d), and $2(\mathrm{e}))$. However, the nanoparticles were distributed throughout the entire depth of the stratum corneum but not into the stratum granulosum. Overall, the two independent qualitative experiments analysing gold-nanoparticle-treated skin, resulted in AuNP signal in the stratum corneum layer with no significant nanoparticle signal in the lower layers.

3.5. En Face MPT-FLIM Intensity Measurement of Gold Nanoparticle Luminescence in Human Viable Skin Depth Study. Having qualitatively assessed the distribution and penetration profile of gold-nanoparticle-treated skin (Figures 1 and 2), the related gold fluorescence signal was then quantified using image software, Image J (National Institutes of Health, Bethesda, Washington, DC, USA) to analyse the number of pixels corresponding to AuNP signal and its depth within the skin (Figure 2(f)). This resulted in detection of a significantly higher concentration of AuNP signal within the stratum corneum in the $10 \mathrm{~nm}$ nanoparticle-treated group compared to the $30 \mathrm{~nm}$ and $60 \mathrm{~nm}$ nanoparticle-treated groups (Figure 2(f)). The AuNP signal below the stratum corneum did not differ significantly from the saline and sodium citrate negative controls. Overall, these data correlates with the qualitative experiments resulting in no significant penetration of the 10,30 , and $60 \mathrm{~nm}$ nanoparticles within the skin.

\subsection{MPT-FLIM Analysis of NAD (P)H after Gold Nanoparticle} Treatment. The metabolic profile of the skin was analysed using MPT-FLIM of total $\mathrm{NAD}(\mathrm{P}) \mathrm{H}$ in the epidermis of excised viable human skin. A depth study involving a series of stacked FLIM images of the treated groups was processed to quantify $\alpha_{1} \% / \alpha_{2} \%$ ratio and total $\mathrm{NAD}(\mathrm{P}) \mathrm{H}$ lifetime intensity. The $\alpha_{1} \% / \alpha_{2} \%$ ratio of $\mathrm{NAD}(\mathrm{P}) \mathrm{H}$ has been reported 
to be inversely related to the metabolic rate [20]. Overall, there was no significant difference of the total $\mathrm{NAD}(\mathrm{P}) \mathrm{H}$ lifetime intensities between any of the groups (Figure 3).

\section{Discussion and Conclusion}

Human skin is regarded as the "gold standard" for skin penetration studies compared to animal model investigations [25]. The present study investigated 10,30 , and $60 \mathrm{~nm}$ gold nanoparticles penetration within excised viable human skin. An en face penetration profile provided gold nanoparticle distribution in excised skin from the stratum corneum to the basale in depth. Analysis of nanoparticle skin penetration involves a number of factors including the skin model, skin barrier integrity, and the inherent physicochemical attributes (size, shape, surface charge, etc.) of the nanoparticles and vehicle of the formulation. To assess the correlation of cell viability (metabolism) and nanoparticle penetration, experiments were conducted with intact human viable skin in an ex vivo diffusion cell system and tested within 24 hours after excision.

It has been previously reported, using spectrophotometry and TEM, that $15 \mathrm{~nm}$ gold nanoparticles suspended in an aqueous solution were capable of penetrating full thickness rat skin [15]. These results are consistent with a recent report by Filon et al. (2011) [14] who showed that $12.9 \mathrm{~nm}$ gold nanoparticles were capable of penetrating into thawed human skin within 24 hours of treatment. However, our study showed that 24-hour treatment of excised viable human skin with 10,30 , and $60 \mathrm{~nm}$ gold nanoparticles resulted in nanoparticle penetration into the stratum corneum only with no significant penetration into the lower layers (Figures 1 and 2). Based on the previous reports showing successful penetration of nanoparticles smaller than $15 \mathrm{~nm}$, it was hypothesised that the $10 \mathrm{~nm}$ nanoparticle group would result in penetration into the viable epidermis. We hypothesise that the lack of penetration observed in our study of the 10,30 , and $60 \mathrm{~nm}$ nanoparticles is associated with the integrity and type of skin sample used (freshly excised viable human skin). As stated, Filon et al. used thawed excised human skin. The freezing and thawing process damaged the viable tissue resulting in increased penetration. We used MPT to detect gold nanoparticle penetration. This is a non-invasive method resulting in minimal disruption of the skin during sample analysis compared to other assays [26] and further emphasises the importance of maintaining skin integrity so as to not bias exposure assessment.

MPT-FLIM was used to assess the effect of gold nanoparticle-penetration on cell metabolism within the viable epidermis. There was no significant change in the total $\mathrm{NAD}(\mathrm{P}) \mathrm{H}$ lifetime intensities for any of the nanoparticle treated groups compared to the negative controls. These results (Figure 3) further support the results shown in Figures 1 and 2 where there was no significant penetration of the nanoparticles below the stratum corneum. Our results show that the stratum corneum protects the viable epidermis by preventing penetration of 10,30 , and $60 \mathrm{~nm}$ gold particles within human skin. When comparing our results to those in literature, we hypothesise that nanoparticle penetration occurs more readily in animal models and nonviable human skin compared to freshly excised viable human skin or in people. Therefore, there should be careful consideration of the animal model and viability of tissue prior to conducting experiments on nanoparticle penetration. This is especially important for experiments analysing the biocompatibility and/or toxicity related to nanoparticle exposure.

\section{Acknowledgments}

Jenny Ordóñez and Dr. Xin Liu are acknowledged for providing valuable laboratory assistance. All members of the TRC and DRC are thanked for their support. This work was supported by The United States Air Force, National Health and Medical Research Council of Australia, EpiDerm, and The University of Queensland Research Scholarship (DCL).

\section{References}

[1] M. S. Roberts, Y. Dancik, T. W. Prow et al., "Non-invasive imaging of skin physiology and percutaneous penetration using fluorescence spectral and lifetime imaging with multiphoton and confocal microscopy," European Journal of Pharmaceutics and Biopharmaceutics, vol. 77, no. 3, pp. 469-488, 2011.

[2] N. A. Monteiro-Riviere and C. L. Tran, Nanotoxicology : Characterization, Dosing and Health Effects, Informa Healthcare, New York, NY, USA, 2007.

[3] X. R. Xia, N. A. Monteiro-Riviere, and J. E. Riviere, "Skin penetration and kinetics of pristine fullerenes $\left(\mathrm{C}_{60}\right)$ topically exposed in industrial organic solvents," Toxicology and Applied Pharmacology, vol. 242, no. 1, pp. 29-37, 2010.

[4] S. Küchler, M. Abdel-Mottaleb, A. Lamprecht, M. R. Radowski, R. Haag, and M. Schäfer-Korting, "Influence of nanocarrier type and size on skin delivery of hydrophilic agents," International Journal of Pharmaceutics, vol. 377, no. 1-2, pp. 169-172, 2009.

[5] J. G. Rouse, J. Yang, J. P. Ryman-Rasmussen, A. R. Barron, and N. A. Monteiro-Riviere, "Effects of mechanical flexion on the penetration of fullerene amino acid-derivatized peptide nanoparticles through skin," Nano Letters, vol. 7, no. 1, pp. 155-160, 2007.

[6] L. J. Mortensen, G. Oberdörster, A. P. Pentland, and L. A. DeLouise, "In vivo skin penetration of quantum dot nanoparticles in the murine model: the effect of UVR," Nano Letters, vol. 8, no. 9, pp. 2779-2787, 2008.

[7] S. A. Coulman, A. Anstey, C. Gateley et al., "Microneedle mediated delivery of nanoparticles into human skin," International Journal of Pharmaceutics, vol. 366, no. 1-2, pp. 190-200, 2009.

[8] H. I. Labouta, D. C. Liu, L. L. Lin et al., "Gold nanoparticle penetration and reduced metabolism in human skin by toluene," Pharmaceutical Research, vol. 28, pp. 2931-2944, 2011.

[9] H. J. Johnston, G. Hutchison, F. M. Christensen, S. Peters, S. Hankin, and V. Stone, "A review of the in vivo and in vitro toxicity of silver and gold particulates: particle attributes and biological mechanisms responsible for the observed toxicity," Critical Reviews in Toxicology, vol. 40, no. 4, pp. 328-346, 2010. 
[10] M. Schneider, F. Stracke, S. Hansen, and U. F. Schaefer, "Nanoparticles and their interactions with the dermal barrier," Dermato-Endocrinology, vol. 1, no. 4, pp. 197-206, 2009.

[11] M. Crosera, M. Bovenzi, G. Maina et al., "Nanoparticle dermal absorption and toxicity: a review of the literature," International Archives of Occupational and Environmental Health, vol. 82, no. 9, pp. 1043-1055, 2009.

[12] G. Cevc and U. Vierl, "Nanotechnology and the transdermal route. A state of the art review and critical appraisal," Journal of Controlled Release, vol. 141, no. 3, pp. 277-299, 2010.

[13] T. W. Prow, J. E. Grice, L. L. Lin et al., "Nanoparticles and microparticles for skin drug delivery," Advanced Drug Delivery Reviews, vol. 63, no. 6, pp. 470-491, 2011.

[14] F. L. Filon, M. Crosera, G. Adami et al., "Human skin penetration of silver nanoparticles through intact and damaged skin," Toxicology, vol. 255, no. 1-2, pp. 33-37, 2009.

[15] G. Sonavane, K. Tomoda, A. Sano, H. Ohshima, H. Terada, and K. Makino, "In vitro permeation of gold nanoparticles through rat skin and rat intestine: effect of particle size," Colloids and Surfaces B, vol. 65, no. 1, pp. 1-10, 2008.

[16] M. S. Roberts, M. J. Roberts, T. A. Robertson et al., "In vitro and in vivo imaging of xenobiotic transport in human skin and in the rat liver," Journal of Biophotonics, vol. 1, no. 6, pp. 478-493, 2008.

[17] M. G. Lin, T. L. Yang, C. T. Chiang et al., "Evaluation of dermal thermal damage by multiphoton autofluorescence and second-harmonic-generation microscopy," Journal of Biomedical Optics, vol. 11, no. 6, Article ID 064006, 2006.

[18] M. G. Lin, W. L. Chen, W. Lo et al., "Three-dimensional skin imaging using the combination of reflected confocal and multiphoton microscopy," in Photonic Therapeutics and Diagnostics III, vol. 6424 of Proceedings of SPIE, p. E4240, San Jose, Calif, USA, January 2007.

[19] M. J. Levene, T. H. Chia, A. Williamson, and D. D. Spencer, "Multiphoton fluorescence lifetime imaging of intrinsic fluorescence in human and rat brain tissue reveals spatially distinct NADH binding," Optics Express, vol. 16, no. 6, pp. 4237-4249, 2008.

[20] L. L. Lin, J. E. Grice, M. K. Butler et al., “Time-correlated single photon counting for simultaneous monitoring of zinc oxide nanoparticles and $\mathrm{NAD}(\mathrm{P}) \mathrm{H}$ in intact and barrier-disrupted volunteer skin," Pharmaceutical Research, vol. 28, pp. 29202930, 2011.

[21] D. L. Kaiser, L. Robert, and J. Watters, National Institute of Standards \& Technology Report of Investigation Reference Material 8011 Gold Nanoparticles, Nominal 10 nm Diameter. $10 \mathrm{p}$.

[22] D. L. Kaiser, L. Robert, and J. Watters, National Institute of Standards \& Technology Report of Investigation Reference Material 8012 Gold Nanoparticles, Nominal 30 nm Diameter. $10 \mathrm{p}$.

[23] D. L. Kaiser, L. Robert, and J. Watters, National Institute of Standards \& Technology Report of Investigation Reference Material 8013 Gold Nanoparticles, Nominal 60 nm Diameter. $10 \mathrm{p}$.

[24] W. Y. Sanchez, T. W. Prow, W. H. Sanchez, J. E. Grice, and M. S. Roberts, "Analysis of the metabolic deterioration of ex vivo skin from ischemic necrosis through the imaging of intracellular $\mathrm{NAD}(\mathrm{P}) \mathrm{H}$ by multiphoton tomography and fluorescence lifetime imaging microscopy," Journal of Biomedical Optics, vol. 15, no. 4, Article ID 046008, 2010.
[25] J. E. Seto, B. E. Polat, R. F. Lopez, D. Blankschtein, and R. F. Langer, "Effects of ultrasound and sodium lauryl sulfate on the transdermal delivery of hydrophilic permeants: Comparative in vitro studies with full-thickness and split-thickness pig and human skin," Journal of Controlled Release, vol. 145, no. 1, pp. 26-32, 2010.

[26] K. König and I. Riemann, "High-resolution multiphoton tomography of human skin with subcellular spatial resolution and picosecond time resolution," Journal of Biomedical Optics, vol. 8, no. 3, pp. 432-439, 2003. 

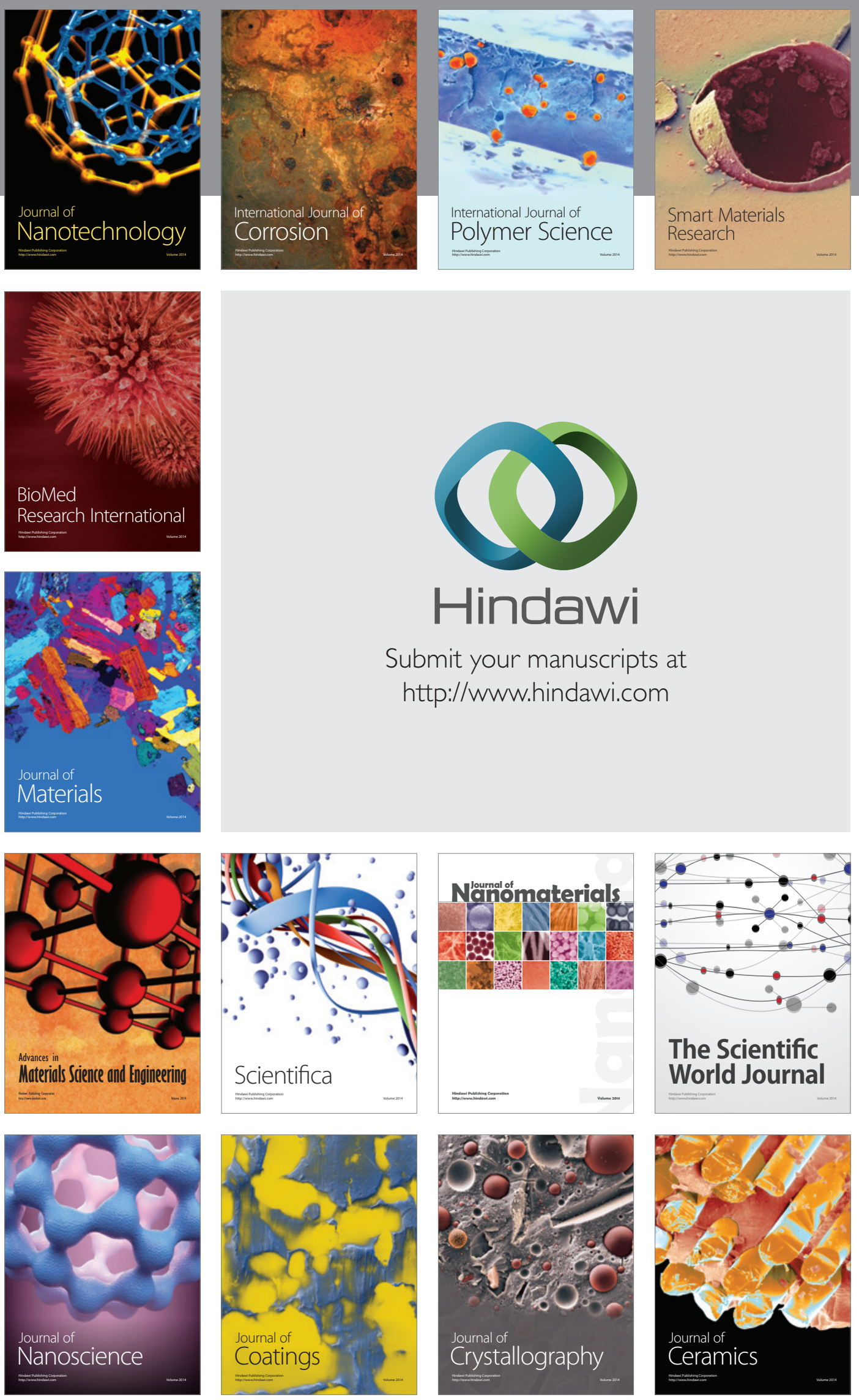

The Scientific World Journal

Submit your manuscripts at

http://www.hindawi.com

\section{World Journal}

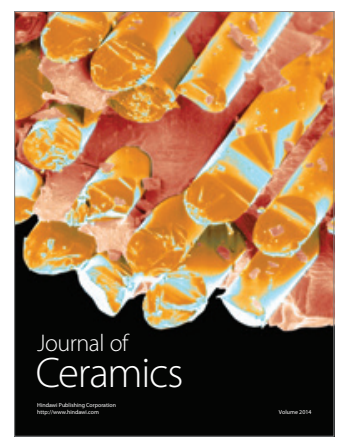

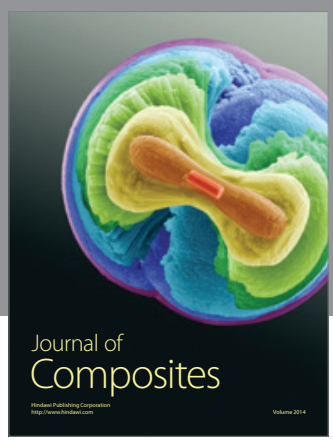
